\title{
Relación de la comunicación familiar y la victimización escolar de adolescentes
}

Karla María Urías Aguirre ${ }^{1}$

\section{Introducción}

En el presente estudio se analiza el papel que juega la comunicación familiar de adolescentes, categorizada en comunicación abierta, comunicación ofensiva y comunicación evitativa, con padre y madre, como variables independientes, en la relación que existe con la victimización escolar (variable dependiente), victimización manifiesta verbal, victimización manifiesta física y victimización relacional. Participaron 1494 adolescentes mexicanos con edades comprendidas entre los 12 y los 18 años. Los resultados obtenidos a partir de los análisis correlacionales indican que la calidad de la comunicación en la familia impacta potenciando o inhibiendo la victimización escolar en los adolescentes.

La violencia escolar es un tema estudiado con mayor ímpetu en los últimos años, ya que antes era visto como algo común que se suscitaba en las aulas. Como se sabe, dentro de la violencia escolar existen varios actores, desde los agresores, pasando por

1 Universidad Autónoma de Sinaloa. 
los espectadores, hasta llegar a las víctimas. El objetivo principal de este trabajo es analizar la victimización escolar en adolescentes y la relación que tiene con la comunicación familiar.

Las condiciones de acoso escolar e intimidación se refieren al alumno que es agredido repetidamente y durante un tiempo por medio de acciones negativas. Estas acciones se describen cuando de manera intencional uno o varios compañeros causan daño, hieren o incomodan a otra persona (Olweus, 2004).

Schwartz (1999) señala la existencia de, al menos, dos subtipos de víctimas de violencia escolar que se diferencian fundamentalmente por su manera de reaccionar ante la situación de violencia (Estévez, Jiménez y Moreno, 2011). La mayor parte de las víctimas se caracteriza por mostrar sumisión y pasividad frente al compañero violento (Schartz, Proctor y Chien, 2001), pero existe un porcentaje menor de víctimas (Olweus, 1978) que muestra un comportamiento hostil y agresivo.

El desarrollo de unas relaciones íntimas, significativas y duraderas (Van Aken y Asendorpf, 1997) depende también de la calidad de las relaciones familiares (Honnes y Robinson, 1993; Sánchez-Queijada y Oliva, 2003). Distintos investigadores señalan que los padres regulan activamente el contexto social del niño y del adolescente, potenciando o inhibiendo su acceso a recursos sociales fuera de la familia (Parke, 2004). De esta forma, los padres influyen de forma positiva o negativa en la elección del grupo de iguales a través del mayor o menor grado de coerción, control y afecto de las prácticas parentales (Engels, Knibbe, De Vries, Drop y Van Breukelen, 1999; Simons, Chao, Conger y Elder, 2001).

Investigaciones sugieren que el ambiente familiar negativo caracterizado por conflictos familiares y bajos niveles de comunicación abierta, afectiva y apoyo es un factor importante para el maltrato entre iguales en la escuela (Gerard y Buehler, 1999; Johnson et al. 2001; Lucia y Breslau, 2006); sin embargo, se sabe poco sobre cómo opera esta influencia. Es probable que el ambiente familiar afecte el ajuste psicosocial de los adolescentes, influyendo la competencia social y sentimientos de soledad (Johnson et al., 2001; Larose y Boivin, 1998; Marturano, Ferrerira 
y Bacarji, 2005) y autoestima (Cava, Musitu y Murgui, 2007; Jiménez, Estévez, Musitu y Murgui, 2007; Musitu y García, 2004). Por otra parte, una relación positiva con los padres, basada en una comunicación abierta, de apoyo, afectiva y sin conflicto podría ayudar a los niños y adolescentes a desarrollar un sentido de seguridad que los alentaría a explorar nuevos contextos sociales (Ainsworth, Blehar, Walters y Wall, 1978; Larose y Boivin, 1998).

Basándonos en estas ideas, este estudio busca conocer el papel de la comunicación familiar en el adolescente y su posible factor protector de la victimización escolar. Específicamente analizaremos la influencia de la comunicación abierta, comunicación ofensiva y comunicación evitativa y su relación con la victimización manifiesta verbal, victimización manifiesta física y victimización relacional.

\section{Método}

Participantes

La muestra se compone por 1494 adolescentes de centros educativos públicos de Sinaloa (nivel secundaria y bachillerato), con edades que abarcan todo el periodo de la adolescencia, desde los 12 a 18 años ( $M=14.8$, D.T. = 1.76). Participantes de ambos sexos (45.4\% varones y $54.6 \%$ mujeres).

\section{Materiales}

Se utilizaron cuestionarios de medidas de comunicación entre padres e hijos y escala de victimización en la escuela. Estos instrumentos han sido adaptados al castellano por el grupo Lisis de la Universidad de Valencia.

Comunicación familiar. Se utilizó el Cuestionario de Evaluación de la Comunicación Familiar (CECF) de Barnes y Olson (1982). Esta escala está compuesta por 20 ítems y dos subescalas que evalúan la comunicación con la madre y la comunicación con el padre. Las respuestas son de tipo Likert y varían de 1 
(nunca) a 5 (siempre). Las dos subescalas presentan una estructura en tres factores: el primero indica el grado de apertura de la comunicación (comunicación abierta, ej.: "Puedo hablarle de lo que pienso sin sentirme mal o incómodo/a"); el segundo indica la presencia de problemas en la comunicación (comunicación ofensiva, ej.: "Me dice cosas que me hacen daño"); y finalmente el tercero indica el grado de evitación de la comunicación familiar (comunicación evitativa, ej.: "No me atrevo a pedirle lo que deseo o quiero").

Victimización escolar. Se utilizó la Escala de Victimización en la Escuela. Equipo Lisis, Universidad de Valencia. Facultad de Psicología (2004). Esta escala está compuesta por 20 ítems. Las respuestas son de tipo Likert y varía de 1 (nunca) a 4 (muchas veces). Presenta una estructura de tres factores, el primero indica el grado de victimización manifiesta verbal (ej.: "Algún compañero/a me ha gritado"); el segundo factor evalúa el grado de victimización manifiesta física (ej.: "Algún compañero/a me ha pegado o golpeado"); y finalmente, el tercer factor evalúa la victimización relacional (ej.: "Algún compañero/a ha contado mentiras sobre mí para que los demás no quieran venir conmigo").

\section{Procedimiento}

Una vez seleccionados los instrumentos, se realizó el contacto con los directivos de los centros escolares. Obtenida la aprobación se hizo una sesión de difusión del proyecto con los profesores y posteriormente se programó la aplicación de la batería de instrumentos.

La aplicación se llevó a cabo por un grupo de investigadores, quienes explicaron a los alumnos el objetivo de la investigación aclarando que su participación sería anónima y voluntaria. El investigador permaneció dentro del aula durante el llenado de los instrumentos para supervisar y aclarar cualquier duda que se suscitara. Se insistió a los adolescentes en el anonimato de la información aportada. Una vez concluidas las respuestas, los alumnos se dirigían al investigador, quien depositaba los instru- 
mentos en un sobre en blanco y cerrado. Este procedimiento fue el mismo para todas las aulas.

Respecto al análisis estadístico, primero se realizó un análisis descriptivo de la muestra con el fin de conocerla a profundidad. Los datos obtenidos de las escalas se procesaron para hacerse un análisis de fiabilidad y tener solidez sobre los datos de interés. Finalmente se hicieron análisis correlacionales de las variables estudiadas con el objetivo de ver la relación existente entre los tipos de comunicación familiar y los tipos de victimización escolar.

\section{Resultados}

Se ha calculado la correlación (producto-momento de Pearson) entre las variables de victimización escolar (victimización manifiesta verbal, victimización manifiesta física y victimización relacional) con las variables de comunicación familiar (comunicación abierta, comunicación ofensiva y comunicación evitativa, con padre y madre). Los resultados indican que las variables independientes del estudio (comunicación con ambos padres) se relacionan significativamente con la variable dependiente (victimización escolar).

La victimización manifiesta verbal se correlaciona positivamente con comunicación ofensiva madre $(r=.197, p<.01)$ y comunicación ofensiva padre $(r=.162, p<.01)$, es decir, a mayor comunicación ofensiva con madre y padre, mayor victimización manifiesta verbal. La victimización manifiesta física se correlaciona positivamente con comunicación ofensiva madre $(r=.147$, $p<.01)$ y comunicación ofensiva padre $(r=.153, p<.01)$, es decir, a mayor comunicación ofensiva con madre y padre, mayor victimización manifiesta física. Finalmente, la victimización relacional se correlaciona con comunicación abierta madre $(r=$ $.119, p<.01)$, comunicación ofensiva madre $(r=.234, p<.01)$ y comunicación ofensiva padre $(r=.170, p<.01)$, es decir, a mayor comunicación abierta madre, menor victimización relacional; a mayor comunicación ofensiva con madre y padre, mayor victimización relacional. 


\section{Discusión}

La comunicación abierta entre padres e hijos facilita que el adolescente se evalúe de modo favorable en los diferentes ámbitos de su vida, mientras que, cuando existen problemas de comunicación, sus percepciones de autoestima familiar y escolar y el apoyo de los miembros de su familia se ven disminuidos (Jiménez, Murgui y Musitu, 2007). En la misma línea de otros autores (Lila y Buelga, 2003; Musitu y García, 2004; Povedano, Hendry, Ramos y Varela, 2011), la percepción del adolescente de un clima familiar positivo, que fomenta la cohesión, el apoyo y la confianza e intimidad entre los miembros de la familia y favorece dinámicas de comunicación abierta y empática, potencia el desarrollo de recursos personales en el adolescente, como la autoestima y la satisfacción con la vida.

Uno de los resultados que se destacan en este estudio y coincidiendo con otras investigaciones (Cava, 2011) es la importancia que la calidad de la comunicación con el padre tiene para las víctimas de acoso escolar. Cava (2011) muestra que los alumnos víctimas de acoso escolar, ya sea verbal, físico o relacional, que tienen una mejor comunicación con sus padres perciben mayor grado de ayuda de sus profesores y muestran una mayor integración en su grupo de iguales y presentan mejor ajuste psicológico que las víctimas de acoso que no cuentan con estos recursos en el ámbito social.

Se infiere que los problemas de comunicación con el contexto familiar pueden convertirse en problemas de ajuste en el contexto escolar, los que, a su vez, influyen negativamente en la salud mental del adolescente (Estévez, Musitu y Herrero, 2005). De esta forma, esta investigación demuestra la relación existente entre la comunicación familiar, ya sea abierta u ofensiva, y la victimización escolar en adolescentes. Esto nos hace tomar en consideración para futuros programas de intervención y prevención donde se trabaje de manera conjunta los contextos no sólo escolares, sino también los familiares. 


\section{Referencias}

Ainsworth, M.D., Blehar, M., Walters, E. y Wall, S. (1978). Patterns of attachment. Hillsdale, NJ: Erlbaum.

Barnes, H. y Olson D. H. (1982). Parent Adolescent Communication Scale. En D.H. Olson, H. McCubbin, H. Barnes, A. Larsen, M. Muxen y W. Wilson (Eds.), Family Inventories: Inventories Used in a National Survey of Families across the Family Life Cycle (pp. 33-48). St. Paul: University of Minnesota Press.

Cava, M. (2011). Familia, profesorado e iguales: Claves para el apoyo a las víctimas de acoso escolar. Psychosocial Intervention. 20(2), 183-192.

Cava, M.J., Musitu, G. y Murgui, S. (2007). Individual and Social Risk Factors Related to Overt Victimization in a Sample of Spanish Adolescents. Psychological Reports, 101, 275-290.

Engels, R.C.M.E., Knibbe, R.A., De Vries, H., Drop, M.J. y Van Breukelen, G.J.P. (1999). Influences of Parental and Best Friends' Smoking and Drinking on Adolescent Use: A Longitudinal Study. Journal of Applied Social Psychology, 29(2), 337-361.

Estévez, E., Jiménez, T. y Moreno, D. (2011). Cuando las víctimas de violencia escolar se convierten en agresores: “¿Quién va a defenderme?". European Journal of Education and Psychology, 3, 177-186.

Estévez, E., Musitu, G. y Herrero, J. (2005). Rol de la comunicación familiar y del ajuste escolar en la salud mental del adolescente. Salud Mental, 28(4), 81-89.

Gerard, J.M. y Buehler, C. (1999). Multiple Risk Factors in the Family Environment and Youth Problem Behaviors. Journal of Marriage and the Family, 61(2), 343-361.

Honess, T. y Robinson, M. (1993). Assesing Parent-Adolescent relationships: A Review of Current Research Issues and Methods. En A.E. Jackson y H. Rodríguez-Tomé (Eds.), Adolescence and Its Social Worlds (pp. 47-66). Hillsdale: Lawrence Erlbaum Associates. 
Jiménez, T., Murgui, S. y Musitu, G. (2007). Comunicación familiar y ánimo depresivo: El papel mediador de los recursos psicosociales del adolescente. Revista Mexicana de Psicología, 24(2), 259-271.

Jiménez, T.I., Estévez, E., Musitu, G. y Murgui, S. (2007). Comunicación familiar y comportamientos delictivos en la adolescencia: El doble rol mediador de la autoestima [Family communication and delinquent behavior in adolescence: the double-mediating role of self-esteem]. Revista Latinoamericana de Psicología, 39(1), 473-485.

Johnson, H.D., LaVoie, J.C. y Mahoney, M. (2001). Interparental Conflict and Family Cohesion Predictors of Loneliness, Social Anxiety, and Social Avoidance in Late Adolescence. Journal of Adolescent Research, 16(3), 304-318.

Larose, S. y Boivin, M. (1998). Attachment to Parents, Social Support Expectations, and Socioemotional Adjustment During the High School-College Transition. Journal of Research on Adolescence, 8(1), 1-27.

Lila, M. S. y Buelga, S. (2003). Familia y adolescencia: el diseño de un programa para la prevención de conductas de riesgo. Encuentros en Psicología Social, 7, 72-78.

Lucia, V.C. y Breslau, N. (2006). Family Cohesion and Children's Behavior Problems: A Longitudinal Investigation. Psychiatry Research, 141(2), 141-149.

Marturano, E.M., Ferrerira, M.D.T. y Bacarji, K.M.G. (2005). An Evalution Scale of Family Environment for Identification of Children at Risk of School Failure. Psychological Reports, 96(2), 307-321.

Musitu G. y García, E. (2004). Consecuencias de la socialización familiar en la cultura española. Psicothema, 16, 288-293.

Olweus, D. (1978). Aggression in the Schools: Bullies and Whipping Boys. Washington, DC: Hemisphere.

Olweus, D. (2004). Conductas de acoso y amenaza entre escolares. $2^{\mathrm{a}}$ Edición. Madrid: Ediciones Morata.

Parke, R.D. (2004). Development in Family. Annual Review of Psychology, 55, 365-399. 
Povedano, A., Hendry, L., Ramos, M. y Varela, R. (2011). Victimización escolar: Clima familiar, autoestima y satisfacción con la vida desde una perspectiva de Género. Psychosocial Intervention, 20(1), 5-12.

Sánchez-Queijada, I. y Oliva, A. (2003). Vínculos de apego con los padres y relaciones con los iguales durante la adolescencia. Revista de Psicología Social, 18(1), 71-86.

Schwartz, D. (1999). Subtypes of Victims and Aggressors in Children's Peer Groups. Journal of Abnormal Child Psychology 28, 181-192.

Schwartz, D., Proctor, L. J. y Chien, D.H. (2001). The Aggressive Victim of Bullying: Emotional and Behavioral Dysregulation as a Pathway to Victimization by Peers. En J. Juvonen y S. Graham (Eds.), Peer harassment in School: The Plight of the Vulnerable and Victimized (pp. 147-174). New York: Guilford Press.

Simons, R. L., Chao, W., Conger, R. D. y Elder, G. H. (2001). Quality of Parenting as Mediator of the Effect of Childhood Defiance on Adolescent Friendship Choices and Delinquency: A Growth Curve Analysis. Journal of Marriage and Family, 63(1), 63-79.

Van Aken, M. A. G. y Asendorpf, J. B. (1997). Support by Parents, Classmates, Friends and Siblings in Preadolescence: Covariation and Compensation Across Relationships. Journal of Social and Personal Relationships, 14(1), 79-93. 
